\title{
Spin exchange of muonium in CdS
}

\author{
J.M. Gil ${ }^{\mathrm{a}, *}$, J. Piroto Duarte ${ }^{\mathrm{a}}$, R.C. Vilão ${ }^{\mathrm{a}}$, H.V. Alberto ${ }^{\mathrm{a}}$, N. Ayres de Campos ${ }^{\text {a }}$, S.F.J. Cox ${ }^{\mathrm{b}}$ \\ ${ }^{\text {a }}$ CEMDRX, Department of Physics, University of Coimbra, P-3004-516 Coimbra, Portugal \\ ${ }^{\mathrm{b}}$ ISIS Facility, Rutherford Appleton Laboratory, Chilton OX11 0QX, UK
}

\section{A R T I C L E I N F O}

Keywords:

Hydrogen in semiconductors

Shallow-donor muonium

Spin exchange

\begin{abstract}
A B S T R A C T
High-statistics, high frequency resolution $\mu$ SR transverse-field time spectra were obtained on a highpurity CdS single crystal in the low temperature range where the shallow-donor muonium state is seen to ionize. A model by Senba was used to fit the data, considering a spin exchange of the muonium electron in the transition region from slow to fast spin-flip regimes. The phase shifts, collapse, and broadening observed in the high-field precession frequencies of the Mu state are explained by this model, implying the involvement of spin dynamics in the ionization process of the shallow-donor muonium state.
\end{abstract}

(c) 2008 Elsevier B.V. All rights reserved.

\section{Introduction}

$\mu \mathrm{SR}$ is a powerful method to study the isolated hydrogen (muonium) states in semiconductors. In particular, the formation and ionization of a muonium $(\mathrm{Mu})$ shallow-donor state in some II-VI semiconductors has been extensively studied [1-4]. Such a state was also found to be formed in InN, GaN and in various oxides [5-7].

In high-purity samples, the spectroscopic signature of this state in transverse fields (TF) and in the high-field regime has the form of a pair of satellite lines of identical amplitude and width, symmetrically placed about the central line corresponding to the diamagnetic muon Larmor precession frequency in the applied TF. Interactions of this state with other shallow donors, native or dopant impurities, have been identified in n-type CdTe, originating a broad line that shows the ionization behavior seen in the initially studied p-type samples [8]. In many other instances, as in the survey of oxides [7], the detectable signal is only a broad line, generally interpreted to correspond to the shallow-donor Mu state due to its disappearance as the temperature is increased with the appropriate energies for the known shallow donors of each material.

In the temperature range of ionization, however, even the cleanest satellite signals, as those obtained in intrinsic CdS, are seen to broaden and move closer to each other as temperature increases, while their combined amplitude is transferred to the central line. The meaning of these spectroscopic changes of the Mu signal and their relation with the ionization process are still not fully understood [9], although these effects are well

\footnotetext{
* Corresponding author. Tel.: +351 239410626; fax: +351829158.

E-mail address: jmgil@ci.uc.pt (J.M. Gil).
}

known from electron spin resonance experiments on the ionization of shallow-donor states [10]. Furthermore, in fits to the TF time spectra using cosines of Lorentzian envelope to describe the satellites, the lines show clear and large phase shifts whose origin needs to be clarified.

Motivated by our inquiries into these matters, Senba has, in one of his last papers before his untimely death, explained in terms of electron spin exchange these features of the ionization of the shallow-donor Mu state [11].

As a tribute to Masa Senba, we present in this work $\mu$ SR results obtained in a single crystal of CdS showing the phase shifts of the satellite lines, together with the results of an analysis with his model. Based on the latter, we draw conclusions regarding the dynamical processes involved in the ionization of the shallowdonor $\mathrm{Mu}$.

\section{Experimental details and data analysis}

We used a nominally undoped CdS single crystal from Eagle-Picher with dimensions of $30 \times 12 \times 1.5 \mathrm{~mm}$. The crystal was cut to have the $c$-axis of the wurtzite unit cell along its longer dimension.

Time-differential $\mu$ SR measurements were performed on the EMU instrument of the ISIS Pulsed Muon Facility during the Training Course in Pulsed Muon Techniques held in February 2005 , using a closed cycle refrigerator and the fly-past set up to minimize the background signal, permitting the analysis of the time spectra to about $22 \mu \mathrm{s}$ after time-zero [12]. The TF measurements were performed at temperatures from $5 \mathrm{~K}$ to room temperature, and at a field of $100 \mathrm{G}$, already in the highfield limit for the extremely low hyperfine interaction of these $\mathrm{Mu}$ states [7-9]. The single crystal was oriented with the c-axis 
perpendicular to the external magnetic field, for which the contributions from all possible orientations of the $\mathrm{Mu}$ anisotropic state average to two lines with a splitting of $A_{h f}=0.229 \mathrm{MHz}$ in the sharpest way possible. The Mu state has its principal axis along the $\mathrm{Cd}-\mathrm{S}$ bonds [1].

The data were analyzed first with three cosine functions of Lorentzian envelope. The second model consists of the sum of a cosine with Lorentzian envelope and a function built from the spin exchange model of Senba [11]. The cosine fits the central line corresponding to the fraction of muons in a diamagnetic state, and the spin-exchange function fits the satellite lines of the paramagnetic Mu state.

The spin-exchange model admits a fittable rate for spin-flip events of the Mu electron. Senba showed in Ref. [11] that the time-dependent muon polarization of a shallow Mu state may be well approximated by

$P(t)=\frac{\mathrm{e}^{-\lambda_{0} t}}{2}\left(\mathrm{e}^{-\mathrm{i}\left[\left(\omega_{12}+\Delta \omega_{0}\right) t+\theta_{0}\right]}+\mathrm{e}^{-\mathrm{i}\left[\left(\omega_{43}-\Delta \omega_{0}\right) t-\theta_{0}\right]}\right)$

where $\lambda_{0}$ is the spin-flip rate dependent relaxation of the polarization, $\Delta \omega_{0}$ and $\theta_{0}$ are its frequency and phase shifts relative to the diamagnetic signal, also spin-flip rate dependent, and $\omega_{12}$ and $\omega_{43}$ are the high-field precession frequencies of the $\mathrm{Mu}$ state. Both the relaxation and the frequency shifts are time dependent, making this model somewhat different from a simple pair of phase shifted and relaxed cosines, although both are approximately constant within the experimental time window of a typical $\mu$ SR experiment for sufficiently small spin-flip rates. The phases of the $\omega_{12}$ and $\omega_{43}$ precessions of the Mu signal are opposite, and increase with the spin-flip rate $\lambda_{S F}$. The frequency shift also increases with $\lambda_{S F}$, giving rise to the collapse of the satellite lines at $\lambda_{S F} \approx 5 A_{h f}$. These features are only visible for muonium states with extremely low hyperfine interactions, since they occur in the transition from slow spin exchange to fast spin exchange, and the transition regime is readily attained for shallow Mu states at very low fields.

The construction of the exact time-domain polarization function, as given by Senba in his paper, involves an infinite series expansion, for which he only states the first 12 terms. That limits the accuracy of the calculation of the time-domain polarization up to the satellite collapse spin-flip rate $\left(\approx 5 A_{h f}\right)$, limiting also the applicability of Senba's model to a temperature that lies at about the middle of the ionization curve.

\section{Results and discussion}

The overall picture of the changes of the spectroscopic signal with temperature is shown in Fig. 1 depicting representative frequency distribution spectra obtained from the time-domain high-statistics, high frequency resolution asymmetry spectra, using a maximum entropy (ME) method.

The main features resulting from fits with the three cosines model to the present data are, similar to the earlier data already mentioned briefly in the Introduction, and corroborating the visual impression from Fig. 1, the collapse and broadening of the satellite lines in the $20-25 \mathrm{~K}$ temperature range, accompanied by a symmetrical shift of the initial phase of the respective cosine functions. Good fits to these new data of high-statistics and high frequency resolution were obtained with all parameters free, but the data close to the range of collapse are still difficult to fit due to the low amplitude of the satellite lines. For clarity of visualization, we have preferred to show in Fig. 2 the remarkable shifts of the initial phases of the Mu precession lines as obtained in fits where the values of the Mu precession frequencies were kept fixed.

The simultaneous transfer of amplitudes between the total paramagnetic signal and the also relaxing diamagnetic central line

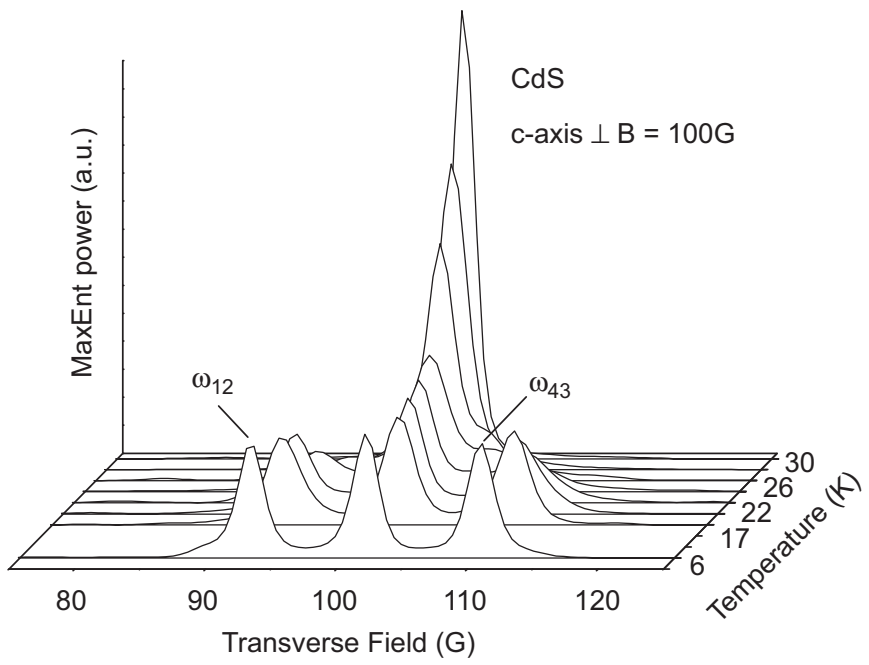

Fig. 1. ME spectra at representative temperatures on a CdS single crystal oriented with the $c$-axis perpendicular to the external TF of $100 \mathrm{G}$.

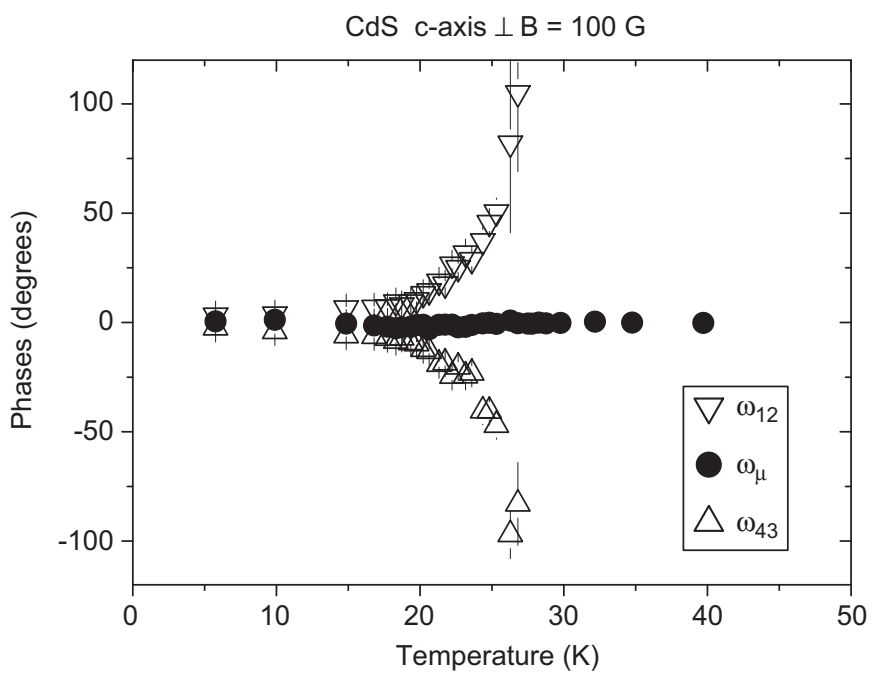

Fig. 2. Temperature variation of the initial phases, from fits to the TF spectra with three cosine functions.

has been taken to identify the process of ionization of the shallowdonor Mu state [9].

The results of fits with the spin exchange model and a cosine function are summarized in Fig. 3. These fits are limited, as explained in Section 2, to spin-flip rates below $1.2 \mu \mathrm{s}^{-1}$, which results in the impossibility of covering the whole temperature range where the ionization process develops.

The time dependence of the paramagnetic fraction is well described by Senba's function for the fitted data, indicating the presence of spin-dynamics along the ionization process, and explaining the phase shifts and collapse of the satellite lines.

The amplitude transfer from the paramagnetic fraction to the diamagnetic fraction is still observed at a temperature identical to that obtained from the fits with the three-line model. A simple formation probability model here was used to fit the varying amplitudes, with the paramagnetic fraction taken to have the following dependence on temperature $T$ :

$f_{\text {param }}=\frac{1}{1+N \exp \left(-\frac{E_{a}}{k T}\right)}$ 


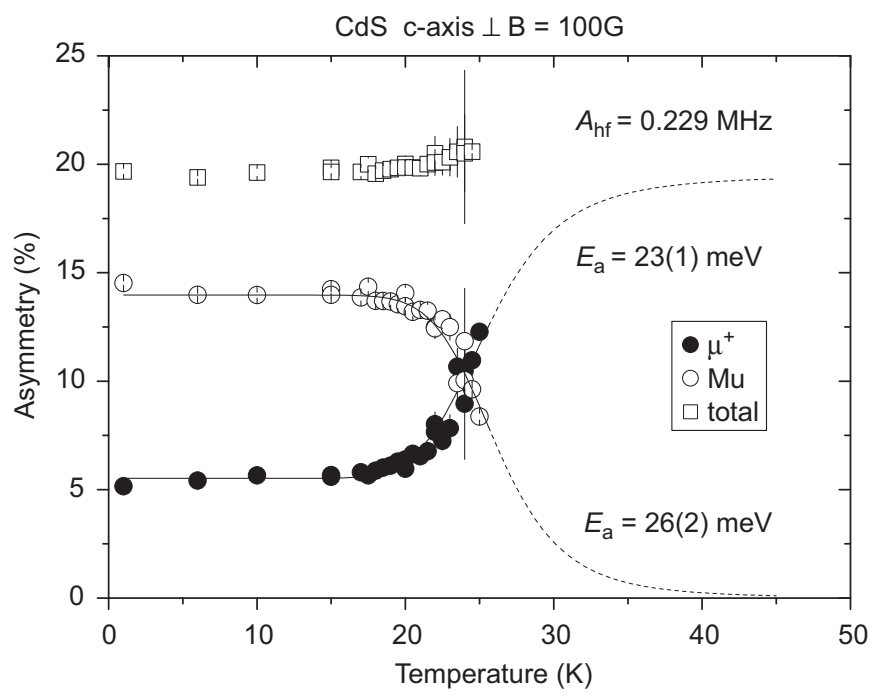

Fig. 3. Temperature variation of the asymmetries fitted with the spin-exchange model (paramagnetic $\mathrm{Mu}$ ) and a cosine function (diamagnetic $\mu^{+}$).

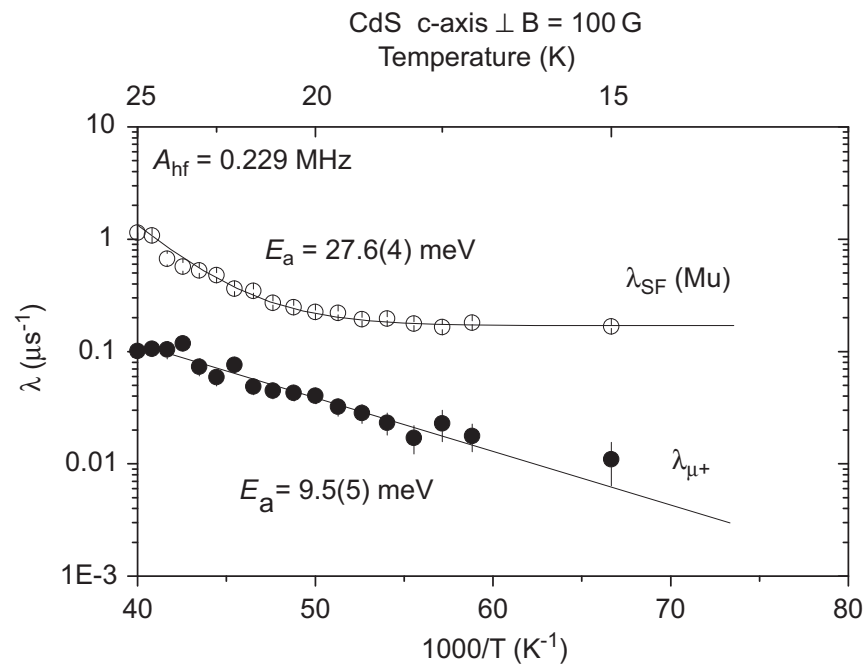

Fig. 4. Arrhenius plot for the paramagnetic spin-flip and the diamagnetic relaxation rates obtained from the fits using Senba's function.

with $N$ being a constant, $k$ the Boltzmann constant, and $E_{a}$ an activation energy. The diamagnetic fraction is the complementary of the paramagnetic fraction. The values obtained for the activation energies are very similar to those obtained earlier and are consistent with the ionization energies of shallow donors in CdS. Nonetheless, one has to be cautious about the values we present here due to the incompleteness of the ionization process within the range analyzed. Indeed, the apparent increase in total amplitude above $20 \mathrm{~K}$ shows that the present model is probably not entirely satisfactory to fit these data. The average phase of the paramagnetic signal remains constant, with a value that is approximately the same as that of the diamagnetic signal.
The spin-flip rate and the diamagnetic line's relaxation become larger as temperature increases, as shown in Fig. 4. The activation energy of the spin-flip rate is again similar to the shallow-donor ionization energy in CdS, but the activation energy of the diamagnetic line's relaxation is considerably lower, which remains to be explained.

A pure spin-flip regime would correspond to scattering processes by conduction electrons from other shallow donors. The transfer of amplitude, in any case, seems to be a separate process from the spin-dynamics if the present model is correct, and may correspond to the intrinsic ionization of the Mu state by its own.

\section{Conclusions}

We have shown that spin-dynamics is involved in the process of ionization of the shallow-donor muonium state in CdS by fitting the spin exchange model of Senba to the paramagnetic Mu TF signal, thus explaining particular features of the data, especially the remarkable shifts of the initial phases of the Mu precession frequencies.

Further improvements are needed in the analysis using Senba's model, namely the computation of more terms of its series, in order to allow a proper description of the data at temperatures above mid-ionization, where the spin-flip rate is larger than the limit imposed by a 12-term series. The energies obtained for the ionization process might then be slightly corrected from the ones obtained here.

A complete picture is still not drawn for the way spindynamics is involved in the ionization process. The present results should be compared with other models for the dynamics of the ionization, namely including separate rates of release and capture of an electron from the conduction band, in a detailed balance, and still considering a spin-flip for each event.

\section{Acknowledgments}

This work was partially supported by the European Commission under the Sixth Framework Programme through the Key Action: Strengthening the European Research Area, Research Infrastructures. Contract no. HII3-CT-2003-505925. The Coimbra team (CEMDRX) was also supported by FCT and FEDER funds under Portuguese contract SFA/2-30.

\section{References}

[1] J.M. Gil, et al., Phys. Rev. Lett. 83 (1999) 5294.

[2] S.F.J. Cox, et al., Phys. Rev. Lett. 86 (2001) 2601.

[3] J.M. Gil, et al., Phys. Rev. B 64 (2001) 075205.

[4] J.S. Lord, et al., J. Phys. Condens. Matter 16 (2004) S4707.

[5] E.A. Davis, et al., Appl. Phys. Lett. 82 (2003) 592

[6] S.F.J. Cox, et al., J. Phys. Condens. Matter 18 (2006) 1079.

[7] K. Shimomura, et al., Phys. Rev. Lett. 92 (2004) 135505.

[8] V. Corregidor, et al., Europhys. Lett. 67 (2004) 247.

[9] S.F.J. Cox, J. Phys. Condens. Matter 15 (2003) R1727.

[10] D.J. Lépine, Phys. Rev. B 2 (1970) 2429.

[11] M. Senba, J. Phys. B At. Mol. Opt. Phys. 38 (2005) 1305

[12] M.C. Lynch, et al., Phys. B 326 (2003) 270. 